\title{
Lumen
}

Selected Proceedings from the Canadian Society for Eighteenth-Century Studies

\section{Le scepticisme de Rousseau dans la Profession de foi du vicaire savoyard}

\section{Marc-André Nadeau}

Volume 25, 2006

URI : https://id.erudit.org/iderudit/1012075ar

DOI : https://doi.org/10.7202/1012075ar

Aller au sommaire du numéro

Éditeur(s)

Canadian Society for Eighteenth-Century Studies / Société canadienne d'étude du dix-huitième siècle

ISSN

1209-3696 (imprimé)

1927-8284 (numérique)

Découvrir la revue

Citer cet article

Nadeau, M.-A. (2006). Le scepticisme de Rousseau dans la Profession de foi du vicaire savoyard. Lumen, 25, 29-40. https://doi.org/10.7202/1012075ar

Copyright (C Canadian Society for Eighteenth-Century Studies / Sociéte canadienne d'étude du dix-huitième siècle, 2006
Ce document est protégé par la loi sur le droit d'auteur. L'utilisation des services d'Érudit (y compris la reproduction) est assujettie à sa politique d'utilisation que vous pouvez consulter en ligne.

https://apropos.erudit.org/fr/usagers/politique-dutilisation/ 


\section{Le scepticisme de Rousseau dans la Profession de foi du vicaire savoyard ${ }^{*}$}

\section{Introduction}

Dans le testament littéraire et philosophique que sont Les Rêveries $d u$ promeneur solitaire, Jean-Jacques Rousseau relate la façon dont il en est venu, à l'âge de quarante ans, à faire une revue complète et critique de ses actions et de ses opinions afin de "fixer une bonne fois pour toute " sa morale et sa métaphysique et, de ce fait, mettre fin aux incertitudes et aux doutes dans lesquels on le jetait sans cesse ${ }^{1}$. "Le résultat de mes pénibles recherches, dit-il, fut tel à peu près que je l'ai consigné depuis dans la Profession de foi du vicaire savoyard, ouvrage indignement prostitué et profané dans la génération présente, mais qui peut faire un jour révolution parmi les hommes si jamais il y renaît du bon sens et de la

* Je tiens à remercier les évaluateurs de la revue Lumen pour leurs commentaires et suggestions ainsi que le Conseil de recherches en sciences humaines du Canada pour son soutien financier.

1 Voir Jean-Jacques Rousseau, Les Rêveries du promeneur solitaire, "Troisième promenade», dans CEuvres complètes, tome I, édition établie par Marcel Raymond et Bernard Gagnebin, Paris, Gallimard, coll. «Bibliothèque de la Pléiade», 1959, p. 1016. Pour Les Rêveries du promeneur solitaire apparaîtront l'abréviation RPS en italiques, la numérotation des "Promenades" en chiffres romains et la pagination des CEuvres complètes, tome I, en chiffres arabes. La numérotation des livres de l'Émile sont en chiffres romains et la pagination des $C E$ uvres complètes, tome $I V$, en chiffres arabes. Pour la Profession de foi du vicaire savoyard, apparaîtront l'abréviation PFVS en italiques et la pagination des CEuvres complètes, tome IV, en chiffres arabes. Pour la Lettre à Christophe de Beaumont, apparaîtront l'abréviation $L C B$ et la pagination des CEuvres complètes, tome $I V$, en chiffres arabes. L'orthographe a été modernisée dans tous les cas. 
bonne foi ${ }^{2}$ ». S'il faut en croire cet extrait, Rousseau aurait énoncé «à peu près " tous les principes de sa morale et de sa métaphysique dans la Profession. Or, un tel renvoi est problématique à plusieurs égards. Non seulement Rousseau maintient-il une certaine distance entre les principes avancés dans la Profession et le contenu entier de sa morale et de sa métaphysique - si la Profession contient à peu près la morale et la métaphysique de Rousseau, il n'est pas dit qu'elle les développe entièrement - mais surtout, il ne précise pas les principes à trouver. Certes, des indications générales sont faites quant à la morale, Rousseau insistant sur la nécessité de combattre l'amour-propre, de renoncer au monde, etc. Cependant, il ne souffle mot de la métaphysique, si ce n'est par la négative dans l'extrait susmentionné. Il signale en effet que la Profession n'a pas été correctement comprise par les autorités philosophiques, politiques et religieuses de son temps et que, dès lors, les prétentions de cet écrit ne sont pas celles que ces autorités lui attribuent.

La réception de la Profession au temps de Rousseau est bien connue. Dès que l'Émile parut en France, il fut interdit et son auteur décrété de prise de corps par les autorités politiques et religieuses. C'est ainsi qu'en octobre 1762, l'archevêque de Paris Christophe de Beaumont publia un Mandement contre l'Émile, et tout particulièrement contre la Profession, "comme contenant une doctrine abominable, propre à renverser la loi naturelle et à détruire les fondements de la religion chrétienne ; [...] comme contenant un très grand nombre de propositions respectivement fausses, scandaleuses, pleines de haine contre l'Église et ses ministres, dérogeantes au respect dû à l'Écriture sainte et à la tradition de l'Église, erronées, impies, blasphématoires et hérétiques ${ }^{3}{ }^{\prime}$. Or, s'il faut en croire la déclaration de Rousseau dans les Rêveries, cette lecture raterait l'essentiel de son propos dans la Profession, sa métaphysique n'étant ni un

2 RPS, III, p. 1018.

3 Christophe de Beaumont, Mandement de Mgr L'Archevêque de Paris, portant condamnation d'un livre qui a pour titre "Émile ou de l'Éducation 》 par Jean-Jacques Rousseau, citoyen de Genève, dans Rousseau, CEuvres, tome III, édition de Michel Launay, Paris, Seuil, 1971, coll. «L'intégrale», p. 336. Voir la réponse de Rousseau dans sa Lettre à Christophe de Beaumont, en particulier aux p. 929-932. Pour une perspective plus large sur ce débat, voir Pierre-Maurice Masson, La Religion de Rousseau, Paris, Hachette, 1916, 3 tomes; Jacques Maritain, Trois Réformateurs : Luther, Descartes, Rousseau, Paris, Plon, 1925 ; et Philippe Lefebvre, Les Pouvoirs de la parole. L'Église et Rousseau (1762-1848), Paris, Cerf, 1992, en particulier les chapitres II («Condamnation parlementaire et censure théologique»), p. 43-66, et III («Juridiction ecclésiastique et droit de condamnation, 1750-1766»), p. 67-84. 
«système d'athéisme», ni des «sentiments d'hypocrisie ${ }^{4} »$. Quelle est donc cette métaphysique que la Profession mettrait en scène mais qui n'aurait pas été correctement comprise par les interprètes contemporains de Rousseau ? Pour tenter de répondre à cette question, nous procéderons en deux temps. Nous tâcherons tout d'abord de dégager la structure globale de ce texte, à savoir ses fondations, son armature, son mouvement et sa finalité. Ensuite, nous nous interrogerons sur les différentes formes de scepticisme que la structure textuelle nous permet de dégager.

\section{I) La structure de la Profession}

La Profession du vicaire savoyard comporte trois parties : tout d'abord le prologue du jeune prosélyte ${ }^{5}$, puis le long récit du vicaire, divisé en deux parties par la seconde intervention du prosélyte.

Le prologue montre que la Profession est faite dans un contexte bien précis - le scepticisme mou du jeune prosélyte, qui incline vers le «scepticisme prétendu» ou le «scepticisme apparent» des philosophes des Lumières ${ }^{6}$ - et dans une intention bien précise, soit le dépassement de ce scepticisme mou. Dans la première partie, le vicaire prend le scepticisme mou pour point de départ, mais il l'approfondit au moyen d'un scepticisme radical et méthodique - ce qu'il appelle son "premier pyrrhonisme ${ }^{7}$. Par l'application de cette méthode sceptique, il découvre quelques évidences (le moi, le monde, etc.) à partir desquelles il déduit une certaine théologie (l'existence d'une volonté intelligente qui meut l'univers et anime la nature) et une certaine morale (la valeur absolue de

4 LCB, p. 963.

5 Émile, IV, p. 559-565.

6 PFVS, p. 568 et p. 632. Sur le scepticisme au XVIII siècle, voir Richard Popkin, Ezequiel de Olaso et Giorgio Tonelli (éds.), Scepticism in the Enlightenment, Dordrecht, Kluwer Academic, 1997 ; Richard Popkin et Johan Van der Zande (éds.), The Skeptical Tradition Around 1800, Dordrecht, Kluwer Academic, 1998 ; Gianni Paganini, Miguel Benitez et James Dybikowski (éds.), Scepticisme, clandestinité et libre pensée, Paris, Honoré Champion, coll. «Libre pensée et littérature clandestine», 2002.

7 PFVS, p. 630. Sur les rapports de Rousseau et du pyrrhonisme, voir Ezequiel de Olaso, "The Two Scepticisms of the Savoyard Vicar», dans Richard A. Watson et James E. Force, The Sceptical Mode in Modern Philosophy: Essays in Honor of Richard H. Popkin, Dordrecht, Martinus Nijhoff Publishers, coll. «Archives internationales d'histoire des idées", 1988, 43-59 ; et Sébastien Charles, Berkeley au siècle des Lumières. Immatérialisme et scepticisme au XVIII siècle, Paris, Vrin, coll. «Histoire de la philosophie», 2003, p. 126-130. 
la conscience pour régler la conduite humaine). Ainsi, par l'application de sa méthode, le vicaire semble parvenir à sortir de son doute initial et à trouver quelques assises philosophiques et morales que le scepticisme ne pourrait révoquer en doute tant elles sont solides.

Or, les premières évidences et les principes théologiques et moraux sont donnés par le vicaire non comme des certitudes claires et distinctes mais comme des croyances probables; non comme des articles de science, mais comme des articles de foi : «Repassant dans mon esprit les diverses opinions qui m'avaient tour à tour entraîné depuis ma naissance, je vis que, bien qu'aucune d'elles ne fût assez évidente pour produire immédiatement la conviction, elles avaient divers degrés de vraisemblance, et que l'assentiment intérieur s'y prêtait ou s'y refusait à différentes mesures ${ }^{8} \gg$. Certes, le cour adhère à ces opinions vraisemblables qu'il sent être vraies. Cependant la raison, autant par défiance d'elle-même que par reconnaissance de l'infinie complexité des questions philosophiques et théologiques qu'elle examine, demeure dans le doute, et principalement en ce qui a trait aux articles de foi, dont le vicaire manifeste à plusieurs reprises le caractère incertain et obscur'. Par conséquent, toute la théologie du vicaire exposée dans la première partie de la Profession reste entachée d'une certaine forme de scepticisme : «Pour être de bonne foi je ne me crois pas infaillible : mes opinions qui me semblent les plus vraies sont peut-être autant de mensonges ; car quel homme ne tient pas aux siennes ? et combien $\mathrm{d}^{\prime}$ hommes sont $\mathrm{d}^{\prime}$ accord en tout ${ }^{10}$ ?» $\mathrm{C}^{\prime}$ est sur un «scepticisme involontaire ${ }^{11}$ » de ce genre que débute $\mathrm{d}^{\prime}$ ailleurs la seconde partie de la Profession - scepticisme que le vicaire veut cette fois que le prosélyte garde à l'esprit tout au long de son examen des religions révélées et des pratiques religieuses. De ce fait, le vicaire vise sans aucun doute à désamorcer toute polémique que pourrait susciter l'acceptation pure et simple de sa critique des révélations. Toutefois, au-delà de ces motifs, il semble que le vicaire soit réellement sincère dans son scepticisme en matière de religion, puisqu'il n'épouse finalement ni tout à fait une religion naturelle ni tout à fait le catholicisme et que, même s'il encourage à la modération et à la tolérance dans la pratique du culte, il reconnaît le bien-fondé d'une certaine intransigeance pour le maintien

8 PFVS, p. 569. Nous soulignons.

9 PFVS, p. 576, 581, 593, 594, 601 et 605.

10 PFVS, p. 605.

11 PFVS, p. 626. 
de l'autorité religieuse, tandis que le narrateur vante quant à lui les mérites du fanatisme dans la longue note qui clôt la Profession ${ }^{12}$.

En somme, l'attitude sceptique quant à la pratique religieuse, à laquelle le vicaire dit en rester à la fin de la seconde partie, ne semble pas si éloignée de l'attitude soutenue discrètement tout au long de la mise en place de sa théologie dans la première partie : «Je n'ai pas la présomption de me croire infaillible ${ }^{13}$ " répète-t-il d'ailleurs à la fin de la seconde partie. Or, si tel est le cas, quelle solution la Profession offre-t-elle au jeune prosélyte, dont le scepticisme nocif est le point de départ et le contexte et qui, jusqu'à la fin de la première partie du moins, maintient ce scepticisme ? Dans quelle mesure la position théorique et pratique du vicaire se distingue-t-elle de celle du prosélyte ? Il semble qu'il faille distinguer ici trois formes différentes de scepticisme ${ }^{14}$.

\section{II) Les différentes formes de scepticisme dans la Profession}

Le vicaire dit en rester finalement à ce qu'il appelle un "scepticisme involontaire ${ }^{15}{ }^{\prime}$. L'expression scepticisme involontaire a ici son importance, car cette forme de scepticisme auquel le vicaire aboutit n'est pas voulu, comme peut l'être celui des philosophes libertins et athées, lesquels affichent un "scepticisme apparent ${ }^{16}$ " qui recèle, ou bien une paresse intellectuelle dogmatique, ou bien une mauvaise foi intéressée et désolante. Le scepticisme final du vicaire n'est pas non plus volontaire comme l'était ce qu'il appelle son "premier pyrrhonisme ${ }^{17}$ ", par lequel il doutait radicalement et méthodiquement de toutes opinions ; pyrrhonisme qui ne peut par nature perdurer dans le temps : "Comment peut-on être sceptique par système et de bonne foi ? je ne saurais le comprendre. Ces philosophes, ou n'existent pas, ou sont les plus malheureux des hommes. Le doute sur les choses qu'il nous importe de connaître est un état trop violent pour l'esprit humain : il n'y résiste pas longtemps ; il se décide

12 PFVS, p. 632-635.

13 PFVS, p. 625.

14 Nous renchérissons ici sur l'analyse d'Ezequiel de Olaso, "The Two Scepticisms of the Savoyard Vicar», op. cit., p. 43-59, qui reconnaît deux formes de scepticisme (scepticisme académicien et scepticisme pyrrhonien) dans la Profession.

15 PFVS, p. 626.

16 PFVS, p. 632.

17 PFVS, p. 631. 
malgré lui de manière ou d'autre, et il aime mieux se tromper que ne rien croire $^{18}{ }^{\prime}$. Contrairement à ce premier pyrrhonisme, le scepticisme involontaire du vicaire ne lui est en effet «nullement pénible», en ce sens qu'il ne paralyse pas son agir moral (qui se satisfait de l'adhésion du cœur et d'opinions vraisemblables) et qu'il semble pouvoir perdurer dans le temps et ainsi constituer une certaine attitude de vie ${ }^{19}$. Même si son scepticisme l'incite à garder «un doute respectueux», à «être toujours modeste et circonspect», à «respecter en silence ce qu'[il] ne saurait ni rejeter, ni comprendre» et surtout à éviter de bouleverser l'ordre public, le vicaire n'en est pas moins en mesure d'être «bien décidé sur les principes de tous [s]es devoirs ${ }^{20} »$. Seulement, il demeure conscient du fait que sa conviction morale est, en définitive, un beau pari ${ }^{21}$.

\section{Le triple scepticisme du vicaire savoyard}

L'examen de la structure de la Profession nous a permis de mettre en lumière une certaine forme de scepticisme dans le contenu de sa théologie et de sa religion ; scepticisme qui s'articule d'une triple façon, à savoir comme «scepticisme apparent», "pyrrhonisme» et «scepticisme involontaire». Si la position finale du vicaire est celle du scepticisme involontaire, il semble cependant qu'une telle position ne puisse être atteinte que par l'intermédiaire des deux autres formes de scepticisme et qu'elle soit donc le fruit d'un mouvement de la pensée et de l'action.

De fait, le premier terme de ce mouvement est le «scepticisme apparent», position incarnée à la fois par le jeune prosélyte désabusé de la religion et de la morale humaines, par les philosophes libertins et athées des Salons parisiens et par le vicaire dans les premiers moments de sa disgrâce. Or un tel scepticisme se révèle, après un examen approfondi, seulement apparent, puisqu'il est ou bien trop mou et superficiel pour être une véritable position morale et philosophique - c'est un tel relativisme qu'adopte vraisemblablement le jeune prosélyte - ou bien trop dogmatique pour pouvoir à bon droit être qualifié de scepticisme : les

19 PFVS, p. 626.

20 PFVS, p. 625-626.

21 Une analyse plus rigoureuse de la nature et de la portée du scepticisme involontaire exposé dans la Profession s'imposerait ; nous n'en connaissons aucune dans la critique rousseauiste. 
philosophes sceptiques-dogmatiques sont certains que la religion est fausse, que la morale est illusoire, que la politique n'est que rapport de forces, etc. Au nom même du scepticisme, une telle attitude morale et philosophique est donc insatisfaisante, et c'est pourquoi un second type de scepticisme s'y oppose, le pyrrhonisme ou le scepticisme radical et méthodique. Celui-ci est purement critique et nullement affirmatif : toute son action consiste à montrer le relativisme et le dogmatisme du premier scepticisme, sans rien proposer en contrepartie. Or ce pyrrhonisme se révèle lui aussi insatisfaisant du fait de la violence de sa remise en question des opinions et des actions - remise en question qui, à la limite, entre en contradiction avec la vie humaine. Une certaine modération dogmatique ou prise en charge relativiste du pyrrhonisme est donc nécessaire pour pouvoir croire quoi que ce soit, ou agir de quelque façon que ce soit.

Le scepticisme apparent et le pyrrhonisme renvoient ainsi à leur opposé au moment même où ils sont épousés ou approfondis. Cependant, dans le mouvement de va-et-vient de l'un à l'autre pôle, une sorte de troisième scepticisme prend place, soit le scepticisme involontaire. Par son argumentation et sa pratique, celui-ci procéderait en effet à la fois à une critique pyrrhonienne du scepticisme apparent et à un adoucissement du pyrrhonisme au nom de l'irréductibilité des opinions et actions vraisemblables auxquelles le cœur adhère malgré ce que la raison peut en penser : «Tel est son scepticisme; et ce scepticisme est bien involontaire, puisqu'il est fondé sur des preuves invincibles de part et $d^{\prime}$ autre, qui forcent la raison de rester en suspens. Ce scepticisme est celui de tout chrétien raisonnable et de bonne foi qui ne veut savoir des choses du ciel que celles qu'il peut comprendre, celles qui importent à sa conduite, et qui rejette avec l'apôtre les questions peu sensées, qui sont sans instruction, et qui n'engendrent que des combats ${ }^{22}$ ».

Il faut bien voir cependant que cette solution n'est possible et durable que si elle n'en est pas une, c'est-à-dire dans la mesure où ce scepticisme demeure involontaire. De fait, dès qu'il n'est plus le produit spontané du mouvement d'oscillation, et qu'il devient une doctrine et une posture, le scepticisme involontaire devient scepticisme apparent. La ligne est donc bien mince entre le scepticisme involontaire du vicaire et le scepticisme apparent du prosélyte et des philosophes athées et libertins. Cela n'implique toutefois pas qu'elle doive être effacée. Au contraire, en forçant un peu le trait, il est possible de distinguer deux attitudes tout à fait 
différentes par rapport à la connaissance, notamment par rapport à la théologie : là où le scepticisme apparent rejette l’Église et la religion comme des formes de supercherie, le scepticisme involontaire du vicaire est non seulement ouvert à une théologie et à une religion naturelles, mais aussi aux dogmes, aux textes et à l'autorité de l'Église catholique : «Appelé dans celle que je professe au service de l'Église, j'y remplis avec toute l'exactitude possible les soins qui me sont prescrits, et ma conscience me reprocherait $d^{\prime} y$ manquer volontairement en quelque point ${ }^{23}$ ».

\section{Le triple scepticisme de la Profession}

À ce triple scepticisme fait vraisemblablement écho la structure tripartite de la Profession, où le prologue montre le «scepticisme apparent» du prosélyte, la première partie l'application du "pyrrhonisme» par le vicaire et la seconde partie le «scepticisme involontaire» où en reste le vicaire. Mais, de manière plus générale, on pourrait dire qu'un triple scepticisme similaire à celui du vicaire est à l'œuvre dans la forme même de la Profession, qui, bien que cela ne soit pas évident, est finalement plus dialogique (ou théâtrale) que discursive : la profession du vicaire est faite à un interlocuteur physiquement présent, à qui les opinions et les conseils du vicaire sont concrètement adressés et qui, en outre, intervient à deux reprises. Nous avons évoqué plus haut l'importance de sa première intervention (le prologue) pour comprendre le contexte, l'intention et la portée de la Profession, mais nous n'avons guère parlé de la seconde, qui fait la jonction entre la première et la seconde partie ${ }^{24}$. Adresse du narrateur à l'interlocuteur - le «cher concitoyen ${ }^{25}$ » - et du jeune prosélyte au vicaire, cette deuxième intervention est précieuse, autant pour la forme que pour le contenu.

Quant à la forme, tout d'abord, elle a le mérite de rappeler et de mettre en évidence celle de la Profession : le narrateur de l'Émile retranscrit l'écrit du narrateur de la Profession, et le narrateur de la Profession retranscrit

23 PFVS, p. 627. Voir aussi $L C B$, p. 956 : «J'avoue bien que la création du monde étant clairement énoncée dans nos traductions de la Genèse, la rejeter positivement serait à cet égard rejeter l'autorité, sinon des Livres Sacrés, au moins des traductions qu'on nous en donne, et c'est aussi ce qui tient le vicaire dans un doute qu'il n'aurait peut-être pas sans cette autorité».

24 PFVS, p. 606.

25 Émile, IV, p. 563. 
quant à lui son dialogue avec le vicaire savoyard. En somme, cette intervention du narrateur souligne à grands traits le dispositif de fiction de la Profession, ce qui semble témoigner d'une certaine distance, au moins entre le narrateur et le vicaire ${ }^{26}$. De ce fait, elle se trouve aussi à inciter le lecteur à prendre lui-même un certain recul par rapport aux propos du vicaire sur la théologie et, peut-être surtout, à se montrer très prudent face aux propos sur la révélation de la seconde partie.

Quant au contenu, cette intervention est également très riche, pour au moins trois raisons. Elle manifeste premièrement que l'adhésion du cœur n'est pas incompatible avec une forme de pyrrhonisme au plan rationnel - «Cependant je voyais des foules d'objections à lui faire...» - et que le pyrrhonisme peut être modéré par l'adhésion elle-même «...je n'en fis pas une, parce qu'elles étaient moins solides qu'embarrassantes, et que la persuasion était pour $\operatorname{lui}^{27}{ }^{2}$. Deuxièmement, elle montre que ce qui séduit le jeune prosélyte, ce n'est pas tant la théologie théiste avancée par le vicaire - «ce que vous dites croire» - que son rapport sceptique à cette théologie - "ce que vous avouez ignorer ${ }^{28}$ ». Autrement dit, c'est l'examen et la recherche du vicaire, recherche ponctuée d'hypothèses, de doutes et d'ignorances avoués, qui le frappent et l'incitent à sortir de son scepticisme apparent. Troisièmement, elle contient un aveu d'ignorance de la part du prosélyte, qui reconnaît la supériorité du vicaire en matière de sagesse et qui est, de ce fait, porté à la réflexion et à l'introspection et désireux d'être instruit par le vicaire.

Prise dans son ensemble, et non pas seulement à partir de la profession du vicaire, la Profession nous semble donc receler une certaine argumentation et pratique sceptiques chez le narrateur, qui font écho à celles du vicaire. Comme le scepticisme involontaire du vicaire, ce scepticisme est en effet issu d'un mouvement de la pensée et de l'action entre le scepticisme apparent (première intervention) et le pyrrhonisme (seconde intervention); il est conciliable avec une certaine adhésion du cœur et il pousse à une recherche ouverte.

26 Sur les dispositifs de fiction à l'œuvre dans la Profession, voir entre autres Michel Coz, La Cène et l'autre scène. Désir et profession de foi chez Jean-Jacques Rousseau, Paris, Honoré Champion, 1998, p. 194-197.

27 PFVS, p. 606.

28 Ibid. 


\section{Le triple scepticisme de Rousseau}

Il nous reste maintenant à voir si un même mécanisme sceptique opère quant au statut de la Profession, à la fois dans l'Émile et dans l'œuvre de Rousseau - ou, pour le dire autrement, si Rousseau lui-même affiche un scepticisme tripartite dans l'usage qu'il fait de la Profession. Ce point est, on le devine, le plus crucial pour résoudre la question du scepticisme de Rousseau. C'est aussi le plus incertain.

On remarquera tout d'abord que, lorsque des commentateurs insistent pour signaler un écart entre la métaphysique du vicaire et celle de Rousseau, c'est le plus souvent pour montrer que le vicaire est plus dogmatique que Rousseau. Par exemple, sur la question de la sociabilité, Allan Bloom fait remarquer : "Il convient de noter ici que le vicaire est bien plus certain de la position spéciale de l'homme dans le Tout ordonné que ne l'est Rousseau lui-même lorsqu'il décrit l'homme dans l'état de nature ${ }^{29} »$. On s'entend donc généralement pour reconnaître à Rousseau un " certain scepticisme ${ }^{30}$ ». Ce scepticisme resterait cependant très limité (critique des sciences et des arts, de l'inégalité, etc.), et serait immédiatement dépassé : Rousseau n'est-il pas le père spirituel ou du romantisme, ou du socialisme, ou du révolutionnarisme, ou du totalitarisme, ou du primitivisme, ou de l'écologisme, ou de l'exhibitionnisme sentimental - en somme, d'une forme ou une autre de doctrine systématique et dogmatique, aux antipodes du scepticisme?

Une telle lecture nous semble toutefois sous-estimer le dispositif de la fiction qui est à l'œuvre partout chez Rousseau, notamment dans la Profession. De fait, c'est le plus souvent par un travail de « fictionnalisation» du monde, des hommes et de lui-même que Rousseau cherche à en comprendre la nature et la portée : «Les lecteurs "me voient dans le pays des chimères ; moi, je les vois dans le pays des préjugés ${ }^{31 " . ~ S o n ~ e f f o r t ~}$

29 Allan Bloom, L'Amour et l'amitié, trad. de Pierre Manent, Paris, Fallois, 1996, p. 81. Voir aussi Léo Strauss, «L'intention de Rousseau», dans Tzvetan Todorov et Gérard Genette (éds.), Pensée de Rousseau, Paris, Seuil, coll. «Points», 1984, p. 90 : «Rousseau [...] avait ce genre de 'tête bien faite' pour laquelle le doute est un 'mol oreiller'».

30 Pierre Burgelin, La philosophie de l'existence de J.-J. Rousseau, Paris, PUF, 1952, coll. «Bibliothèque de philosophie contemporaine», p. 42 : «Lecteur de Montaigne, il professe un certain scepticisme en face de la tradition philosophique ". Voir aussi Robert Derathé, Le Rationalisme de Jean-Jacques Rousseau, Paris, PUF, 1948, p. 41-45 ; et Yvon Belaval, "Rationalisme sceptique et dogmatisme du sentiment chez Jean-Jacques Rousseau», Annales de la société J.-J. Rousseau, 38 (1969), p. 7-24.

31 Émile, IV, p. 549. 
consiste à arracher ces lecteurs à la prison de ce qui est observable. Se méfiant des chimères, ils sont incapables de quitter le pays des préjugés et de s'ouvrir à ce qui constitue la finalité idéale de leur existence, c'està-dire ce que Rousseau appelle la nature ${ }^{32} »$. Ainsi, les grandes fictions rousseauistes, comme l'état de nature du Second Discours, la constitution idéale du Contrat social, l'éducation parfaite de l'Émile, voire le récit de sa propre vie dans Les Confessions, ne seraient pas tant les piliers d'un système dogmatique qu'une façon de mener une recherche proprement philosophique (et donc sceptique) sur la nature des choses. En dernière instance, Rousseau reconnaîtrait que «leur vérité est en suspens ${ }^{33}$ ».

Comme le vicaire et le narrateur de la Profession, Rousseau semble donc approcher l'être, le monde et les hommes selon un mouvement d'oscillation allant du dogmatisme de la fiction à la remise en question de la fiction ; mouvement par lequel l'adhésion est rendue possible et la recherche stimulée.

\section{Conclusion}

Nous aimerions cependant évoquer une difficulté importante, avec laquelle cette hypothèse du scepticisme de Rousseau est aux prises. La nature du scepticisme involontaire que nous avons dégagé chez Rousseau apparaît en effet problématique pour trois raisons au moins.

Premièrement, pour être un véritable scepticisme et non un "scepticisme apparent», celui-ci doit procéder d'un mouvement d'oscillation entre le dogmatisme et le pyrrhonisme. Or, si le pyrrhonisme est une méthode plutôt qu'une position véritablement prise au sérieux méthode qui, en outre, est quelque peu laissée en plan à la fin de la Profession $^{34}$ — dans quelle mesure ce nécessaire mouvement de la pensée peut-il encore prendre place?

Deuxièmement, nous l'avons vu, le scepticisme involontaire autorise l'adhésion du cœur aux opinions et actions vraisemblables — autant que

32 Philip Knee, La Parole incertaine: Montaigne en dialogue, Québec, Presses de l’Université Laval, coll. «République des Lettres», 2003, p. 131-132.

33 Ibid., p. 135.

34 Voir PFVS, p. 630-631 : “J'ai pris mon parti : je m'y tiens ; ma conscience est tranquille, mon cœur est content. [...] Je resterai comme je suis, de peur qu'insensiblement le goût de la contemplation, devenant une passion oiseuse, ne m'attiédît sur l'exercice de mes devoirs, et de peur de retomber dans mon premier pyrrhonisme, sans retrouver la force d'en sortir». 
cette vraisemblance reste visible aux yeux de la raison. Or, le consentement rationnel à cette adhésion dogmatique du cœur est difficilement conciliable avec la nature même du scepticisme. Comment en effet déterminer des degrés d'incertitude si ce n'est à partir d'un critère qui transcende le scepticisme lui-même ? Comment fixer des limites au scepticisme sans le perdre du même souffle ${ }^{35}$ ?

Enfin, troisièmement, même si «l'expérience de l'incertitude se trouve au cœur de sa démarche, elle n'y est pas thématisée. Elle est au contraire voilée $[\ldots]^{36}$ ». Rousseau demeure très discret sur la question de son scepticisme, de sorte que nous ne pouvons nous empêcher d'en questionner la valeur, la nature et la portée. Rousseau est-il finalement un philosophe sceptique au même titre que Montaigne, par exemple?

Il n'y a évidemment pas de réponses claires et nettes à ces différents problèmes de l'œuvre rousseauiste. Toutefois, cette absence de réponses ne signifie pas que Rousseau n'y ait guère songé, car toute sa pensée témoigne d'une profonde acuité d'esprit sur les enjeux philosophiques les plus pointus. Au contraire, une telle absence nous paraît porteuse de sens sur la nature et la portée du scepticisme de Rousseau, qui, en quelque sorte, renvoie de ce fait à une figure de la philosophie à demi tracée, à demi visible. C'est dire que l'examen du scepticisme de Rousseau dans la Profession ouvre sur une plus vaste étude, qui reste à faire, de l'ensemble de la pensée et de la place que le scepticisme y occupe.

\section{MARC-ANDRÉ NADEAU Université d'Ottawa}

35 Ce problème est exprimé de façon particulièrement éloquente par Montaigne dans $l^{\prime}$ 'Apologie de Raymond Sebond" : «Cette propension à une proposition plutôt qu'à une autre, qu'est-ce autre chose que la reconnaissance de quelque plus apparente vérité en celle-ci qu'en celle-là ? Si notre entendement est capable de la forme, des linéaments, du port et du visage, de la vérité, il la verrait entière, aussi bien que demie, naissante, et imparfaite. Cette apparence de vérisimilitude qui les fait prendre plutôt à gauche qu'à droite, augmentez-la ; cette once de vérisimilitude qui incline la balance, multipliez-la de cent, de mille onces, il en adviendra enfin que la balance prendra parti tout à fait, et arrêtera un choix et une vérité entière. Mais comment se laissent ils plier à la vraisemblance, s'ils ne connaissent le vrai ? Comment connaissent ils la semblance de ce de quoi ils ne connaissent pas l'essence? Ou nous pouvons juger tout à fait, ou tout à fait nous ne le pouvons pas». (Essais, éd. Pierre Villey, Paris, PUF, coll. «Quadrige», 1992, livre II, ch. 12, p. 560-561.) 